OPEN ACCESS

Edited by:

Christopher J. Martyniuk,

University of Florida, United States

Reviewed by:

Yuwei Xie,

University of Saskatchewan, Canada

Alexis Maria Wormington,

University of Florida, United States

*Correspondence:

Sébastien Duperron

sebastien.duperron@mnhn.fr

Specialty section: This article was submitted to

Environmental Health,

a section of the journal

Frontiers in Public Health

Received: 03 April 2020

Accepted: 09 July 2020

Published: 21 August 2020

Citation:

Duperron S, Halary S, Gallet A and Marie B (2020) Microbiome-Aware

Ecotoxicology of Organisms:

Relevance, Pitfalls, and Challenges.

Front. Public Health 8:407.

doi: 10.3389/fpubh.2020.00407

\section{Microbiome-Aware Ecotoxicology of Organisms: Relevance, Pitfalls, and Challenges}

\author{
Sébastien Duperron ${ }^{1,2 *}$, Sébastien Halary ${ }^{1}$, Alison Gallet ${ }^{1}$ and Benjamin Marie ${ }^{1}$ \\ ${ }^{1}$ Muséum National d'Histoire Naturelle, CNRS, UMR7245 Mécanismes de Communication et Adaptation des \\ Micro-organismes, Paris, France, ${ }^{2}$ Institut Universitaire de France, Paris, France
}

Over the last 15 years, the advent of high-throughput "omics" techniques has revealed the multiple roles and interactions occurring among hosts, their microbial partners and their environment. This microbiome revolution has radically changed our views of biology, evolution, and individuality. Sitting at the interface between a host and its environment, the microbiome is a relevant yet understudied compartment for ecotoxicology research. Various recent works confirm that the microbiome reacts to and interacts with contaminants, with consequences for hosts and ecosystems. In this paper, we thus advocate for the development of a "microbiome-aware ecotoxicology" of organisms. We emphasize its relevance and discuss important conceptual and technical pitfalls associated with study design and interpretation. We identify topics such as functionality, quantification, temporality, resilience, interactions, and prediction as major challenges and promising venues for microbiome research applied to ecotoxicology.

Keywords: toxicology, microbiota, symbiosis, contaminants, resilience, environment

\section{INTRODUCTION: THE MICROBIOME IS RELEVANT TO ECOTOXICOLOGY}

The significance of microbes to multicellular organisms is long documented. Because only a fraction of microorganisms can be isolated in culture, it is the advent of high-throughput sequencing technologies which ultimately revealed how diverse and numerically abundant they were. Microorganisms form complex symbiotic communities of eukaryotes, bacteria, archaea, and viruses referred to as the microbiome (1-3). Over the last 15 years, the microbiome has been a new frontier in Life Sciences (4), and microorganisms were shown to be involved and even necessary in many host functions including nutrition, defense, immunity, development, and behavior $(2,5,6)$. A current paradigm assumes that most animals and plants harbor a microbiome (7). However, some species of comb jellies and nematomorpha, as well as certain life stages of insects including honeybee larvae, are apparently devoid of a microbiome, suggesting that this may be an oversimplification $(8,9)$. In humans, the microbiome may represent as many cells as the hosts and up to 1,000 times more genes, questioning the concept of individuality, and the limits of self $(10,11)$. The holobiont concept, referring to the entity formed by a host organism and its various microbial associates, arouse to encompass the complexity of hosts and their microbiome $(12,13)$. All these discoveries have fueled a "microbiome revolution" that increasingly spreads through all fields of life sciences, with extensions to behavioral and human sciences $(14,15)$. 
Recent research focuses mostly on the links between hosts and their microbiome, and the reciprocal influence they exert on each other, revealing its significance to host physiology, homeostasis, disease, health, and fitness (16). Interestingly, most members of the microbiome are located on epithelia (mucosa, skin...), i.e., animal or plant polarized tissues that separate the inside from the outside of the organism. Sitting at the interface between a host and its environment, epithelia and their associated microbiome are the hosts buffer and first line of defense against contaminants and environmental stressors (17). Deep-sea hydrothermal vent mussels are an example of this. They harbor bacteria located in the gill epithelium that oxidize hydrogen sulfide, a compound toxic to their hosts, and fix carbon that contributes hosts nutrition (18). Toxicology studies investigating the effects of chemical compounds on organisms examine the accumulation, bio-transformation, elimination, and effects in tissues, and are thus beginning to account for associated microbiome. This paper aims to emphasize the relevance, pitfalls, and promises of hostassociated microbiome research for ecotoxicology and advocates for the emergence of a "microbiome-aware ecotoxicology" of multicellular organisms, i.e., an approach that fully incorporates the microbiome compartment as a dynamic interface interacting with host and environment (Figure 1).

\section{THE MICROBIOME RESPONDS TO AND INTERACTS WITH CONTAMINANTS}

Data on effects of environmental contaminants on microbiomes has been published over the last years, with a focus on animal gut-associated bacteria (19-22). Published studies include controlled exposure of model organisms to various types of contaminants including pesticides, antibiotics, heavy metals, xenobiotics, or nanoparticles $(23,24)$. Bacterial community composition is typically assessed using the $16 \mathrm{~S}$ rRNA sequence as a taxonomic marker that identifies Operational Taxonomic Units (OTUs), a commonly used proxy for species. The effect of exposures on OTU richness and diversity is then evaluated using multivariate statistics to test whether contaminants interfere with microbiome composition $(25,26)$. Many studies include complementary analyses on host parameters of toxicological relevance such as markers of the immune system, tissue histology, and developmental markers.

Besides descriptive studies that correlate exposures and microbiome variations, functional studies directly investigate the interactions between the gut microbiome and contaminants [Figure 2; (27)]. It has been shown that microbiomes of mammals guts are able to metabolize a wide range of xenobiotics (e.g., polycyclic aromatic hydrocarbons, polychlorobiphenyls, and nitrotoluenes) and could protect animals from deleterious effects (19). But the microbiome may also activate some compounds and mediate toxicity to hosts. For instance, the human colon microbiome was shown to convert polycyclic aromatic hydrocarbons into estrogenic metabolites with consequences on hormonal equilibrium (28); and the nephrotoxicity of melamine in rats results from its conversion into toxic cyanuric acid mediated by the bacterium Klebsiella terrigena (29). Many pharmaceutical drugs such as lovastatin or loperamide were also proved to be activated in the small intestine by bacteria-mediated biotransformations (30,31). Alternatively, xenobiotics can also alter activities of the gut microbiome (23). Besides the obvious example of antibiotics, many molecules such as epoxiconazole or glyphosate, both pesticides, are known to induce shifts in microbiome compositions (32). Because the microbiome substantially responds and interacts with contaminants, it must now be considered a key player in toxicology.

\section{PRODUCING AND INTERPRETING MICROBIOME DATA RELEVANT TO ECOTOXICOLOGY}

\section{Conceptual Pitfalls: Identifying a "Good" Microbiome and a "Good" Model Species}

Ecotoxicology studies that address the microbiome rely on a microbial ecology background and need to consider the caveats associated with this discipline. Experimental investigations to date have focused mostly on bacteria. However, numerous studies have demonstrated the significance of Archaea and microbial Eukaryotes, including fungi, to host physiology $(33,34)$, and the key role of phages in regulating bacterial populations (35, 36). A comprehensive description of microbiome functioning requires all components to be accounted for (37). However, the lack of universal, easy-to-obtain markers for some groups, notably for viruses, still precludes the development of systematic analyses that require deep metagenomics, and specific expert analysis pipelines.

Besides, OTUs composition provides only a partial description of the real microbial diversity. Indeed, a single $16 \mathrm{~S}$ rRNAbased OTU can encompass a diversity of distinct bacterial genotypes, potentially quite different in term of their respective functional phenotypes and responses to a stimulus (38, 39). The genus Vibrio for example includes strains with very different lifestyles, including commensals, light-producing mutualists of the squid Euprymna scolopes, and pathogens of numerous metazoans, that all display almost identical $16 \mathrm{~S}$ rRNA sequences (40). The relative abundances and dynamics of these different phenotypes, can thus not be monitored using $16 \mathrm{~S}$ rRNA.

Another major difficulty is the general lack of baseline knowledge regarding microbiomes of toxicology model species, for which very little-to-no data is available regarding wild populations (41-43). Besides, organisms used in tests are often sourced from rearing facilities, and have thus experienced domestication, a process documented to lead to massive changes in bacterial microbiome compositions. In vertebrates, changes include overall bacterial species richness decrease and shift in taxa abundances due to dietary, social, and environmental conditions of captivity [reviewed in $(42,44)$ ]. Effects in other taxa are less documented and less clear-cut. In the silkworm for example, domestication is associated with higher bacterial diversity (45). The representativity of model species in ecotoxicology vs. their wild relatives thus 


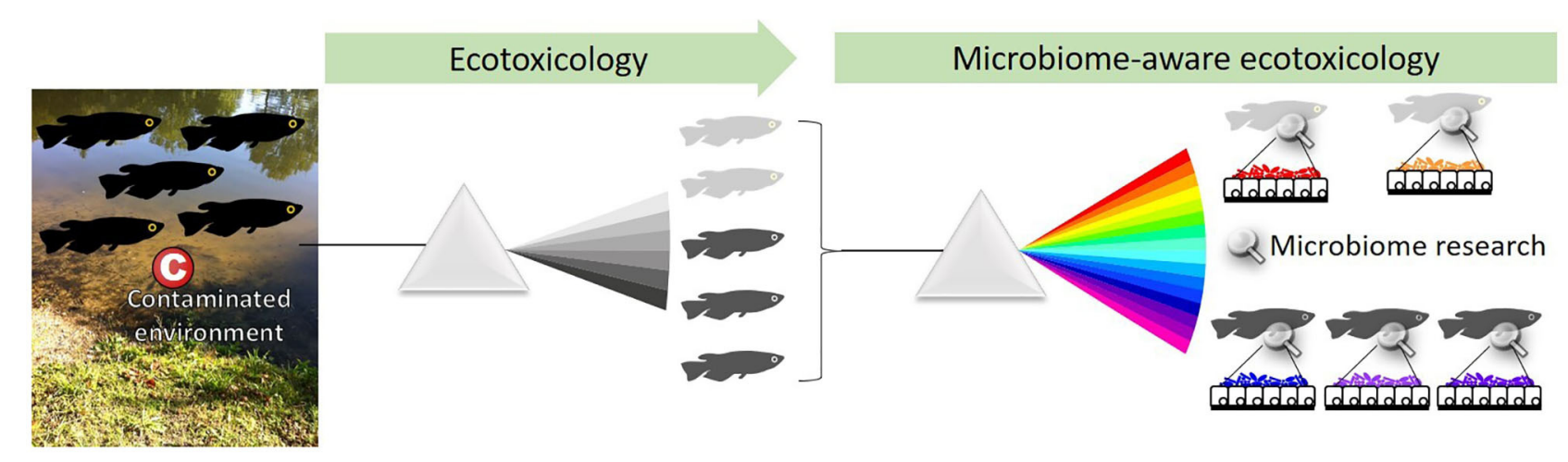

FIGURE 1 | Ecotoxicology studies the effect of chemicals on organisms at the population level. A microbiome-aware ecotoxicology perspective acknowledges the importance of associated microorganisms in their hosts biology, at the level of individuals as well as populations. Indeed, the microbiome is interacting with hosts, environment, and contaminants and may be linked with health status (here, light vs. dark gray). The microbiome thus needs to be integrated as an element of the system, and protocols to investigate ecotoxicological effects at each level need to be adapted.

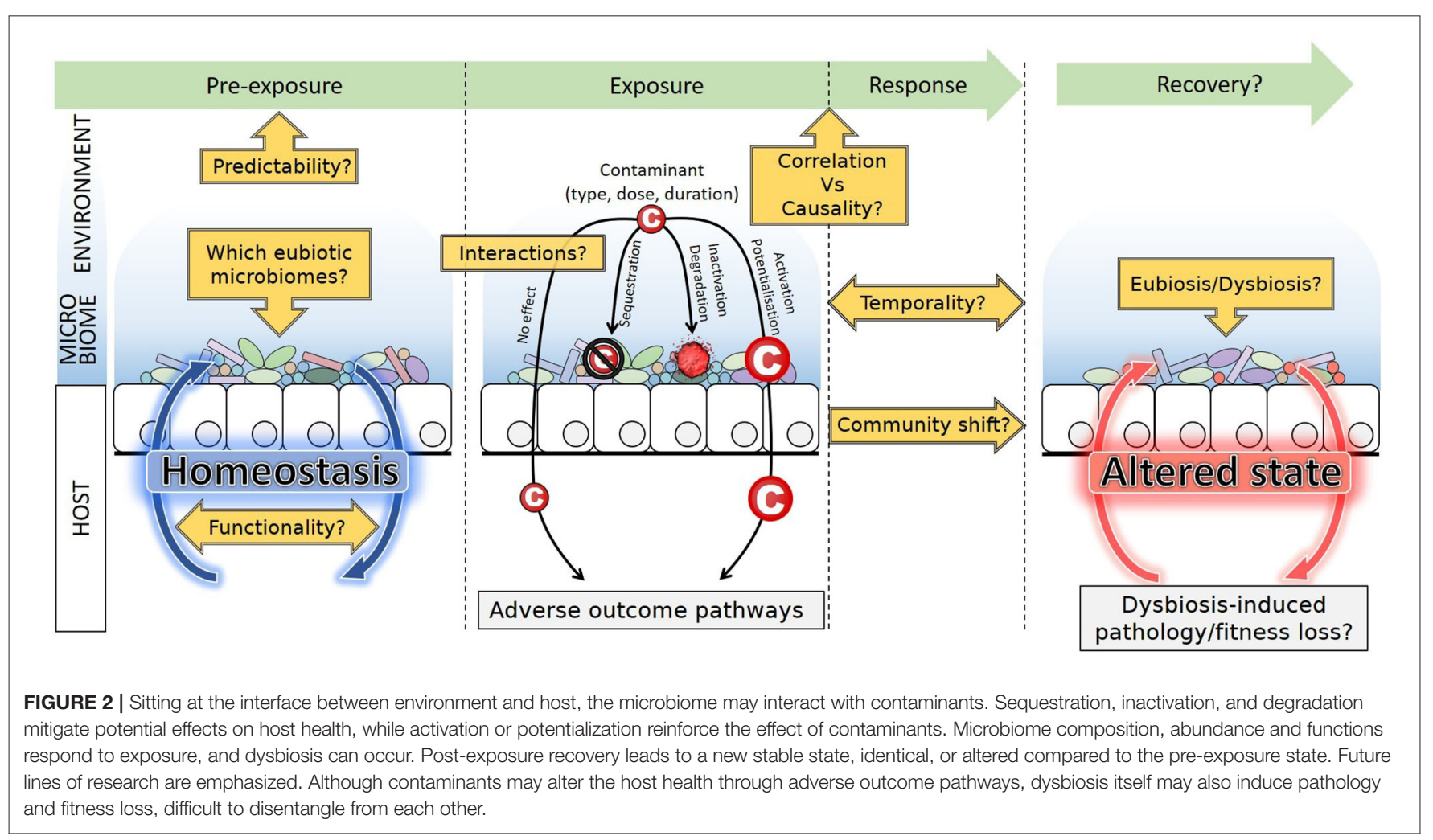

remains to be evaluated in the light of their domestication history. Interestingly, humans are no exception to this trend, and gut microbiomes in industrialized societies greatly differ from the recent ancestral microbiome and from that of contemporary traditional populations (e.g., hunters-gatherers). Changes in diets, sanitation, and medical practices have led to a functional shift from fiber to mucus degraders, high frequency of antibiotic resistance, loss of particular taxa (e.g., Spirochaetes), and overall diversity decrease $(46,47)$. This is assumed to result in non-optimal microbiomes associated with increased risk of chronic diseases.
Lack of knowledge, along with inter-individual variability (discussed below Technical Pitfalls: Performing the Right Experiment to Detect Effects), undermines the identification of the "normal," balanced microbiome composition, i.e., the eubiotic state. This compromises the proper diagnosis of a dysbiosis (an "abnormal," unbalanced) state upon exposure to contaminants (Figure 2). Indeed, although many factors can cause dysbiosis that may lead to health issues, it is not easy to establish what a healthy/eubiotic microbiome is (48). Recently, authors insisted that dysbiosis due to stressors is first of all the destabilization of the stable eubiotic state. Changes 
in abundances of certain beneficial taxa are evident signs of dysbiosis, but interestingly, increased inter-individual variability in microbiome composition could be another signature of dysbiosis (49).

In between the relative simplicity of most invertebrateassociated microbiomes in which a few OTUs are usually dominant [e.g., (50)] and the extreme complexity of mammalassociated microbiomes (with hundreds to thousands of OTUs), teleost fish and their tens to a few hundred bacterial OTUs offer an interesting intermediate, besides their relevance to the monitoring of aquatic ecosystems (51). Choosing a model thus involves addressing different levels of microbiome complexity, functionality, and domestication history. Whether current models in toxicology are relevant to microbiome-aware ecotoxicology studies needs to be evaluated.

\section{Technical Pitfalls: Performing the Right Experiment to Detect Effects}

Most studies using controlled microcosms are monitoring various compartments that are potential sources of microbial diversity (e.g., food for animals, water for aquatic organisms). When scaling up to more holistic approaches such as mesocosms or the natural environment, potential sources of microorganisms dramatically increase, requiring the investigation of additional compartments (e.g., food, prays, parasites, water, particles, sediments).

Fifteen years of human gut microbiome research revealed the high level of intra- (between body regions or life stages) and inter-individual heterogeneity in community compositions $(1,52)$. Although less documented, high levels of intra- and inter-individual variation are reported in other taxa including fish [e.g., Atlantic cod, salmon, rainbow trout, zebrafish, $(26,51,53-55)]$. In the zebrafish for example, gut-associated communities become increasingly different from those in the environment, and inter-individual variation increases across development (56). Skin-associated communities are different on different body regions in the rainbow trout (55). These examples emphasize the importance of replication levels, and of addressing the exact same life stage and tissue region in all individuals.

Sex-differentiated responses to compounds are commonly reported in ecotoxicology studies, for example in medaka fish exposed to cyanotoxins (57). Sex also influences microbiome composition in various vertebrates, probably due to hormones and sex-specific immunity responses (58). It also affects microbiome responses. In lab-reared stickleback fed different diets, diet induced changes in some bacterial taxa abundances, but effects on bacteria in males were uncorrelated with effects observed in females, supporting that diet effects were clearly sex-specific (59). Authors measured similar sex-specific diet effects in mice and humans. Sex-specific effects on microbiome responses to contaminants are also documented. Exposure to silver nanoparticles was for example shown to modify the gut microbiome structure in male zebrafish, but not in females (60). Experimental design should thus carefully consider confounding factors of which sex is an important one.

\section{THE ROADS LESS TRAVELED: CHALLENGES IN MICROBIOME-AWARE ECOTOXICOLOGY}

\section{Functionality and Integration}

One major finding of the Human Microbiome Project was that despite high levels of intra- and inter-individual variation in the taxonomic compositions of bacterial communities, the functions they performed, as encoded by the metagenome, were highly conserved (1). Similar functions are thus performed by taxonomically distinct microorganisms. This concept known as functional redundancy is now recognized as key to the resistance and resilience of microbial communities $(61,62)$. Because of this, and the fact that closely related bacteria can display markedly different functionalities, community composition is not a reliable predictor of functions. Predictive tools for functional profiling based on composition [e.g., PICRUSt; (63)] thus suffer limitations, and identity and functions should ideally be investigated in tandem. Functional capabilities can be evaluated through metagenomic sequencing, but genes and functions that are expressed at specific time points are better evaluated by metatranscriptomic or metaproteomic approaches. Metabolomics, which map metabolites, are another important tool that profiles ongoing metabolisms, and thus informs functions $(64,65)$ although, as for all of the above, the improvement of databases supporting metabolite identifications will be critical (66). The integration of these approaches in multi-omics appears challenging, yet particularly promising for revealing the causal role of the microbiome and mechanisms involved in contaminants metabolism and toxic effects $(67,68)$. This will be key in integrating the microbiome in adverse outcome pathways (AOPs) and risk assessment [Figure 2; (22)].

\section{Quantification}

Microbiome-aware ecotoxicology should identify contaminant threshold values relevant to microbiomes. Indeed, microbial communities may shift rapidly and non-linearly between contrasting alternative, more or less stable states provided some parameters reach threshold values. The existence of yetundescribed tipping points is for example hypothesized to explain the existence of bimodal distributions of abundances of certain bacteria in the human gut $(69,70)$, and may be a trigger of dysbiosis. To identify tipping points in ecotoxicology, studies should examine dose-dependent responses and chronic exposure to low doses as done for toxicological effect on host traits, for example the determination of non-observable adverse effect limit (NOAEL).

Microbiome composition assessments also need to become more quantitative. Indeed, metabarcoding datasets produce taxa relative abundances tables, and their variations. In these, an increase in one group thus cannot be properly interpreted, as it may as well represent a lower decrease relative to other groups in a globally shrinking population. Bacterial densities in guts of distinct lineages of rainforest ants were for example shown to vary by orders of magnitude based on qPCR quantifications; interestingly, absolute abundance variations were better correlated with habitat (arboreal or terrestrial) and trophic position than actual community compositions (71). 
Antibiotics, which are reported to affect relative abundances in bacterial communities, act first by affecting the total number of bacteria present, as was clearly demonstrated for streptomycin and sancomycin, this being their major influence on the microbiome $(72,73)$. Absolute abundances are relevant to our understanding of the environment-host-microbiome continuum, and should thus be informed whenever possible, for example using quantitative PCR (74). Quantifying bacteria within organisms is however challenging, as demonstrated by the very different estimations of bacteria-to-human cell ratios found in the literature (75).

\section{Temporality and Resilience}

The nature and amplitude of variations are important aspects of microbiome response to contaminants. Composition of communities and diversity indices are still the main endpoints of most studies. However, the dynamics of these variations during and after exposure are certainly as important. In humans, microbiome dynamics are individual-dependent (76). Dynamics inform resilience, evaluating whether variations have longterm effects on the microbiome, or whether it fully recovers and returns to a naive, pre-exposure stable state (Figure 2). Antibiotic exposure was for example shown to affect human gut bacterial communities for several months post-exposure, and similar effects can be expected with many contaminants (77, 78). Whether iterative exposure to some contaminants may lead to habituation, and thus become less influential to microbiomes over time, is also an important issue. Finally, how microbiome resilience itself may be affected by environmental factors (e.g., temperature, $\mathrm{pH}$, interactions, seasonality) remains to be investigated.

\section{Interactions and Prediction}

The holobiont is more than just the sum of its parts (13). With dozens-to-thousands distinct coexisting microbial taxa, and many more if phages are considered, an animal's gut or skin is a whole ecosystem in which multiple interactions among members and with the host influence its functioning. These as well as interactions with the environment, including the contaminants and microorganisms occurring there, need to be accounted for. For example, a new method coupling spatial imaging of metabolites and bacterial genotypes, MetaFISH, was developed to characterize interactions occurring between symbiotic bacteria and the gill epithelial cells of hydrothermal vent mussels. It allowed the identification of metabolites located at the hostsymbiont interface on tissue sections at the micrometer scale (79). Such a method is promising to monitor small-scale interactions between contaminants, the microbiome and the host and further explore causality (68). Co-occurrence networks that are based on positive or negative correlations between the occurrence of microorganisms, functions, and environmental parameters also help in exploring interactions and formulating hypotheses [reviewed in (80)]. A strong relationship between the presence of a contaminant and that of certain bacterial taxa can suggest an ability to metabolize the former, which can then be tested (19).
Changes in the network structure itself can indicate microbial successions in time series experiments or dysbiosis, and may support modeling approaches $(81,82)$.

\section{CONCLUSION: WHAT CAN THE MICROBIOME DO FOR ECOTOXICOLOGY AND VICE VERSA?}

By analogy with the famous essay written by Dobzhansky (83), it is tempting these days to suggest that "Nothing in Biology makes sense except in the light of the microbiome." Ecotoxicology is no exception to this trend, and must not lag behind other disciplines that have embraced the microbiome revolution. However, the microbiome is not just another ecotoxicological endpoint, but a peculiar and complex biological compartment that exhibits its own ecological, metabolic, functional, and thus ecotoxicological rules (26). Instead, a microbiome-aware ecotoxicology of organisms needs to develop (Figure 1). This involves questioning, and not only transferring, classical toxicology protocols and model organisms' relevance to microbiome studies. Close cooperation between microbial ecologists and ecotoxicologists is needed. They have a lot in common: the complexity of microbiomes and their responses mirrors that of contaminants and their interactions; and both domains start with reductionist approaches, and strive to scale up to holistic approaches that encompass ecosystems full complexity and produce real-life-relevant data.

A major challenge is to move on from observing correlations to addressing causality, and ultimately explain processes, e.g., demonstrate mitigating effects of the microbiome at the population level in a given ecosystem. Repeatability is a key point, which involves inter-studies comparisons and metaanalyses for which tools are becoming available [e.g., Amplicon Sequence Variants for OTU clustering; (84)]. Microbiome features including taxa or functions may become bioindicators of contamination, as recently proposed in stream ecosystems (85). Modeling interactions between environment, contaminants, microbiomes, and hosts will become tractable, with a certain level of predictive power (86). No doubt the dialogue between disciplines will result in mutual enrichment, and will allow to make the most of the microbiome revolution applied to ecotoxicology.

\section{AUTHOR CONTRIBUTIONS}

$\mathrm{SD}, \mathrm{SH}, \mathrm{AG}$, and $\mathrm{BM}$ have contributed to the writing of the manuscript and the production of figures. All authors have read and approved the final version.

\section{ACKNOWLEDGMENTS}

We thank O. Adamovsky, C. Bernard, Y. Desdevises, and C. Duval for fruitful discussions dealing with the various topics covered herein. 


\section{REFERENCES}

1. The Human Microbiome Project Consortium. Structure, function and diversity of the healthy human microbiome. Nature. (2012) 486:20714. doi: $10.1038 /$ nature11234

2. McFall-Ngai M, Hadfield MG, Bosch TCG, Carey HV, DomazetLoso $\mathrm{T}$, Douglas AE, et al. Animals in a bacterial world, a new imperative for the life sciences. Proc Natl Acad Sci USA. (2013) 110:3229-36. doi: 10.1073/pnas.1218525110

3. Tipton L, Darcy JL, Hynson NA. A developing symbiosis: enabling crosstalk between ecologists and microbiome scientists. Front Microbiol. (2019) 10:292. doi: 10.3389/fmicb.2019.00292

4. Alivisatos AP, Blaser MJ, Brodie EL, Chun M, Dangl JL, Donohue TJ, et al. A unified initiative to harness Earth's microbiomes. Science. (2015) 350:5078. doi: 10.1126/science.aac8480

5. Rakoff-Nahoum S, Paglino J, Eslami-Varzaneh F, Edberg S, Medzhitov R. Recognition of commensal microflora by toll-like receptors is required for intestinal homeostasis. Cell. (2004) 118:229-41. doi: 10.1016/j.cell.2004.07.002

6. Archie EA, Theis KR. Animal behaviour meets microbial ecology. Anim Behav. (2011) 82:425-36. doi: 10.1016/j.anbehav.2011.05.029

7. Rosenberg E, Zilber-Rosenberg I. The hologenome concept of evolution after 10 years. Microbiome. (2018) 6:78. doi: 10.1186/s40168-018-0457-9

8. Martinson VG, Moy J, Moran NA. Establishment of characteristic gut bacteria during development of the honeybee worker. Appl Environ Microbiol. (2012) 78:2830-40. doi: 10.1128/AEM.07810-11

9. Hammer TJ, Sanders JG, Fierer N. Not all animals need a microbiome. FEMS Microbiol Lett. (2019) 366:fnz117. doi: 10.1093/femsle/fnz117

10. Gilbert SF, Sapp J, Tauber AI. A symbiotic view of life: we have never been individuals. Q Rev Biol. (2012) 87:325-41. doi: 10.1086/668166

11. Rees T, Bosch T, Douglas AE. How the microbiome challenges our concept of self. PLoS Biol. (2018) 16:e2005358. doi: 10.1371/journal.pbio.2005358

12. Mindell DP. Phylogenetic consequences of symbioses: eukarya and eubacteria are not monophyletic taxa. Biosystems. (1992) 27:53-62. doi: 10.1016/0303-2647(92)90046-2

13. Bordenstein SR, Theis KR. Host biology in light of the microbiome: ten principles of holobionts and hologenomes. PLoS Biol. (2015) 13:e1002226. doi: 10.1371/journal.pbio.1002226

14. Blaser MJ. The microbiome revolution. J Clin Invest. (2014) 124:41625. doi: 10.1172/JCI78366

15. Cryan JF, Dinan TG. Talking about a microbiome revolution. Nat Microbiol. (2019) 4:552-3. doi: 10.1038/s41564-019-0422-9

16. Selber-Hnatiw S, Rukundo B, Ahmadi M, Akoubi H, Al-Bizri H, Aliu AF, et al. Human gut microbiota: toward an ecology of disease. Front Microbiol. (2017) 8:1265. doi: $10.3389 /$ fmicb.2017.01265

17. Barr JJ, Auro R, Furlan M, Whiteson KL, Erb ML, Pogliano J, et al. Bacteriophage adhering to mucus provide a non-host-derived immunity. Proc Natl Acad Sci USA. (2013) 110:10771-6. doi: 10.1073/pnas.1305923110

18. Halary S, Riou V, Gaill F, Boudier T, Duperron S. 3D FISH for the quantification of methane- and sulphur-oxidising endosymbionts in bacteriocytes of the hydrothermal vent mussel Bathymodiolus azoricus. ISME J. (2008) 2:284-92. doi: 10.1038/ismej.20 08.3

19. Claus SP, Guillou H, Ellero-Simatos S. The gut microbiota: a major player in the toxicity of environmental pollutants? Npj Biofilms Microbiomes. (2016) 2:16003. doi: 10.1038/npjbiofilms.2016.3

20. Jin Y, Wu S, Zeng Z, Fu Z. Effects of environmental pollutants on gut microbiota. Environ Pollut. (2017) 222:1-9. doi: 10.1016/j.envpol.2016.11.045

21. Rosenfeld CS. Gut dysbiosis in animals due to environmental chemical exposures. Front Cell Infect Microbiol. (2017) 7:396. doi: 10.3389/fcimb.2017.00396

22. Adamovsky O, Buerger AN, Wormington AM, Ector N, Griffitt RJ, Bisesi $\mathrm{JH}$, et al. The gut microbiome and aquatic toxicology: an emerging concept for environmental health. Environ Toxicol Chem. (2018) 37:275875. doi: 10.1002/etc.4249

23. Licht TR, Bahl MI. Impact of the gut microbiota on chemical risk assessment. Curr Opin Toxicol. (2019) 15:109-13. doi: 10.1016/j.cotox.2018.09.004
24. Monroy-Torres R, Hernández-Luna MA, Ramírez-Gómez XS, López-Briones $\mathrm{S}$. Role of the microbiome as the first metal detoxification mechanism. Prebiot Probiot Potent Benef Nutr. Health. (2019). doi: 10.5772/intechopen.89232

25. Ramette A. Multivariate analyses in microbial ecology. FEMS Microbiol Ecol. (2007) 62:142-60. doi: 10.1111/j.1574-6941.2007.00375.x

26. Evariste L, Barret M, Mottier A, Mouchet F, Gauthier L, Pinelli E. Gut microbiota of aquatic organisms: a key endpoint for ecotoxicological studies. Environ. Pollut. Barking Essex. (2019) 248:989-99. doi: 10.1016/j.envpol.2019.02.101

27. Schmidt R, Ulanova D, Wick LY, Bode HB, Garbeva P. Microbe-driven chemical ecology: past, present and future. ISME J. (2019) 13:265663. doi: 10.1038/s41396-019-0469-x

28. Van de Wiele T, Vanhaecke L, Boeckaert C, Peru K, Headley J, Verstraete W, et al. Human colon microbiota transform polycyclic aromatic hydrocarbons to estrogenic metabolites. Environ Health Perspect. (2005) 113:6-10. doi: 10.1289/ehp.7259

29. Zheng X, Zhao A, Xie G, Chi Y, Zhao L, Li H, et al. Melamine-induced renal toxicity is mediated by the gut microbiota. Sci Transl Med. (2013) 5:172ra22. doi: 10.1126/scitranslmed.3005114

30. Lavrijsen K, van Dyck D, van Houdt J, Hendrickx J, Monbaliu J, Woestenborghs R, et al. Reduction of the prodrug loperamide oxide to its active drug loperamide in the gut of rats, dogs, and humans. Drug Metab Dispos. (1995) 23:354-62.

31. Yoo D-H, Kim IS, Van Le TK, Jung I-H, Yoo HH, Kim D-H. Gut microbiotamediated drug interactions between lovastatin and antibiotics. Drug Metab Dispos. (2014) 42:1508-13. doi: 10.1124/dmd.114.058354

32. Xu C, Liu Q, Huan F, Qu J, Liu W, Gu A, et al. Changes in gut microbiota may be early signs of liver toxicity induced by epoxiconazole in rats. Chemotherapy. (2014) 60:135-42. doi: 10.1159/000371837

33. Hoffmann C, Dollive S, Grunberg S, Chen J, Li H, Wu GD, et al. Archaea and fungi of the human gut microbiome: correlations with diet and bacterial residents. PLoS ONE. (2013) 8:e66019. doi: 10.1371/journal.pone.0066019

34. Richard ML, Sokol H. The gut mycobiota: insights into analysis, environmental interactions and role in gastrointestinal diseases. Nat Rev Gastroenterol Hepatol. (2019) 16:331-45. doi: 10.1038/s41575-019-0121-2

35. Mirzaei MK, Maurice CF. Ménage à trois in the human gut: interactions between host, bacteria and phages. Nat Rev Microbiol. (2017) 15:397408. doi: 10.1038/nrmicro.2017.30

36. Bettarel Y, Halary S, Auguet J-C, Mai TC, Van Bui N, Bouvier T, et al. Corallivory and the microbial debacle in two branching scleractinians. ISME J. (2018) 12:1109-26. doi: 10.1038/s41396-017-0033-5

37. Rowan-Nash AD, Korry BJ, Mylonakis E, Belenky P. Cross-domain and viral interactions in the microbiome. Microbiol Mol Biol Rev MMBR. (2019) 83:e00044-18. doi: 10.1128/MMBR.00044-18

38. Andam CP. Clonal yet different: understanding the causes of genomic heterogeneity in microbial species and impacts on public health. mSystems. (2019) 4:e00097-19. doi: 10.1128/mSystems.00097-19

39. Hanafiah A, Lopes BS. Genetic diversity and virulence characteristics of Helicobacter pylori. isolates in different human ethnic groups. Infect Genet Evol. (2020) 78:104135. doi: 10.1016/j.meegid.2019.104135

40. Sawabe T, Kita-Tsukamoto K, Thompson FL. Inferring the evolutionary history of vibrios by means of multilocus sequence analysis. J Bacteriol. (2007) 189:7932-6. doi: 10.1128/JB.00693-07

41. Uenishi G, Fujita S, Ohashi G, Kato A, Yamauchi S, Matsuzawa T, et al. Molecular analyses of the intestinal microbiota of chimpanzees in the wild and in captivity. Am J Primatol. (2007) 69:367-76. doi: 10.1002/ajp.20351

42. Hird SM. Evolutionary biology needs wild microbiomes. Front Microbiol. (2017) 8:725. doi: 10.3389/fmicb.2017.00725

43. Shinohara A, Nohara M, Kondo Y, Jogahara T, Nagura-Kato GA, Izawa M, et al. Comparison of the gut microbiotas of laboratory and wild Asian house shrews (Suncus murinus) based on cloned 16S rRNA sequences. Exp Anim. (2019) 68:531-9. doi: 10.1538/expanim.19-0021

44. Alessandri G, Milani C, Mancabelli L, Mangifesta M, Lugli GA, Viappiani A, et al. The impact of human-facilitated selection on the gut microbiota of domesticated mammals. FEMS Microbiol Ecol. (2019) 95:fiz121. doi: 10.1093/femsec/fiz121 
45. Chen B, Du K, Sun C, Vimalanathan A, Liang X, Li Y, et al. Gut bacterial and fungal communities of the domesticated silkworm (Bombyx mori) and wild mulberry-feeding relatives. Isme J. (2018) 12:225262. doi: 10.1038/s41396-018-0174-1

46. Blaser MJ. Antibiotic use and its consequences for the normal microbiome. Science. (2016) 352:544-5. doi: 10.1126/science.aad9358

47. Sonnenburg ED, Sonnenburg JL. The ancestral and industrialized gut microbiota and implications for human health. Nat Rev Microbiol. (2019) 17:383-90. doi: 10.1038/s41579-019-0191-8

48. Iebba V, Totino V, Gagliardi A, Santangelo F, Cacciotti F, Trancassini M, et al. Eubiosis and dysbiosis: the two sides of the microbiota. New Microbiol. (2016) 39:1-12.

49. Zaneveld JR, McMinds R, Thurber RV. Stress and stability: applying the Anna Karenina principle to animal microbiomes. Nat Microbiol. (2017) 2:17121. doi: 10.1038/nmicrobiol.2017.121

50. Raymann K, Shaffer Z, Moran NA. Antibiotic exposure perturbs the gut microbiota and elevates mortality in honeybees. Plos Biol. (2017) 15:e2001861. doi: 10.1371/journal.pbio.20 01861

51. Llewellyn MS, Boutin S, Hoseinifar SH, Derome N. Teleost microbiomes: the state of the art in their characterization, manipulation and importance in aquaculture and fisheries. Front Microbiol. (2014) 5:207. doi: 10.3389/fmicb.2014.00207

52. Rothschild D, Weissbrod O, Barkan E, Kurilshikov A, Korem T, Zeevi D, et al. Environment dominates over host genetics in shaping human gut microbiota. Nature. (2018) 555:210-5. doi: 10.1038/nature 25973

53. Star B, Haverkamp THA, Jentoft S, Jakobsen KS. Next generation sequencing shows high variation of the intestinal microbial species composition in Atlantic cod caught at a single location. BMC Microbiol. (2013) 13:248. doi: 10.1186/1471-2180-13-248

54. Duperron S, Halary S, Habiballah M, Gallet A, Huet H, Duval C, et al. Response of fish gut microbiota to toxin-containing cyanobacterial extracts: a microcosm study on the medaka (Oryzias latipes). Environ Sci Technol Lett. (2019) 6:341-7. doi: 10.1021/acs.estlett.9b00297

55. Lowrey L, Woodhams DC, Tacchi L, Salinas I. Topographical mapping of the rainbow trout (Oncorhynchus mykiss) microbiome reveals a diverse bacterial community with antifungal properties in the skin. Appl Environ Microbiol. (2015) 81:6915-25. doi: 10.1128/AEM.01826-15

56. Stephens WZ, Burns AR, Stagaman K, Wong S, Rawls JF, Guillemin K, et al. The composition of the zebrafish intestinal microbial community varies across development. Isme J. (2016) 10:644-54. doi: 10.1038/ismej.20 15.140

57. Le Manach S, Khenfech N, Huet H, Qiao Q, Duval C, Marie A, et al. Genderspecific toxicological effects of chronic exposure to pure microcystin-LR or complex Microcystis aeruginosa extracts on adult medaka fish. Environ Sci Technol. (2016) 50:8324-34. doi: 10.1021/acs.est.6b01903

58. Haro C, Rangel-Zúñiga OA, Alcalá-Díaz JF, Gómez-Delgado F, Pérez-Martínez P, Delgado-Lista J, et al. Intestinal microbiota is influenced by gender and body mass index. PLoS ONE. (2016) 11:e0154090. doi: 10.1371/journal.pone.01 54090

59. Bolnick DI, Snowberg LK, Hirsch PE, Lauber CL, Org E, Parks B, et al. Individual diet has sex-dependent effects on vertebrate gut microbiota. Nat Commun. (2014) 5:4500. doi: 10.1038/ncomms5500

60. Ma Y, Song L, Lei Y, Jia P, Lu C, Wu J, et al. Sex dependent effects of silver nanoparticles on the zebrafish gut microbiota. Environ Sci Nano. (2018) 5:740-51. doi: 10.1039/C7EN00740J

61. Allison SD, Martiny JBH. Colloquium paper: resistance, resilience, and redundancy in microbial communities. Proc Natl Acad Sci USA. (2008) 105(Suppl. 1):11512-9. doi: 10.1073/pnas.0801925105

62. Moya A, Ferrer M. Functional redundancy-induced stability of gut microbiota subjected to disturbance. Trends Microbiol. (2016) 24:402-13. doi: 10.1016/j.tim.2016. 02.002

63. Langille MGI, Zaneveld J, Caporaso JG, McDonald D, Knights D, Reyes JA, et al. Predictive functional profiling of microbial communities using 16S rRNA marker gene sequences. Nat Biotechnol. (2013) 31:81421. doi: $10.1038 /$ nbt. 2676

64. Bundy JG, Davey MP, Viant MR. Environmental metabolomics: a critical review and future perspectives. Metabolomics. (2009) 5:3. doi: 10.1007/s11306-008-0152-0

65. Gao B, Chi L, Mahbub R, Bian X, Tu P, Ru H, et al. Multiomics reveals that lead exposure disturbs gut microbiome development, key metabolites, and metabolic pathways. Chem Res Toxicol. (2017) 30:996-1005. doi: 10.1021/acs.chemrestox.6b 00401

66. Gertsman I, Barshop BA. Promises and pitfalls of untargeted metabolomics. J Inherit Metab Dis. (2018) 41:355-66. doi: 10.1007/s10545-017-0130-7

67. Rohart F, Gautier B, Singh A, Lê Cao KA. mixOmics: an R package for'omics feature selection and multiple data integration. PLoS Comput. Biol. (2017) 13:e1005752. doi: 10.1371/journal.pcbi.1005752

68. Koh A, Bäckhed F. From association to causality: the role of the gut microbiota and its functional products on host metabolism. Mol Cell. (2020) 78:584-96. doi: 10.1016/j.molcel.2020. 03.005

69. Lahti L, Salojärvi J, Salonen A, Scheffer M, de Vos WM. Tipping elements in the human intestinal ecosystem. Nat Commun. (2014) 5:4344. doi: $10.1038 /$ ncomms5344

70. van Nes EH, Arani BMS, Staal A, van der Bolt B, Flores BM, Bathiany S, et al. What do you mean, "tipping point"? Trends Ecol Evol. (2016) 31:9024. doi: 10.1016/j.tree.2016.09.011

71. Sanders JG, Lukasik P, Frederickson ME, Russell JA, Koga R, Knight R, et al. Dramatic differences in gut bacterial densities correlate with diet and habitat in rainforest ants. Integr Comp Biol. (2017) 57:705-22. doi: 10.1093/icb/icx088

72. Willing BP, Russell SL, Finlay BB. Shifting the balance: antibiotic effects on host-microbiota mutualism. Nat Rev Microbiol. (2011) 9:23343. doi: $10.1038 /$ nrmicro 2536

73. Vieira-Silva S, Sabino J, Valles-Colomer M, Falony G, Kathagen G, Caenepeel $\mathrm{C}$, et al. Quantitative microbiome profiling disentangles inflammationand bile duct obstruction-associated microbiota alterations across PSC/IBD diagnoses. Nat Microbiol. (2019) 4:1826-31. doi: 10.1038/s41564-0190483-9

74. Tkacz A, Hortala M, Poole PS. Absolute quantitation of microbiota abundance in environmental samples. Microbiome. (2018) 6:110. doi: 10.1186/s40168-018-0491-7

75. Sender R, Fuchs S, Milo R. Are we really vastly outnumbered? Revisiting the ratio of bacterial to host cells in humans. Cell. (2016) 164:33740. doi: 10.1016/j.cell.2016.01.013

76. Flores GE, Caporaso JG, Henley JB, Rideout JR, Domogala D, Chase J, et al. Temporal variability is a personalized feature of the human microbiome. Genome Biol. (2014) 15:531. doi: 10.1186/s13059-014-0531-y

77. Dethlefsen L, Relman DA. Incomplete recovery and individualized responses of the human distal gut microbiota to repeated antibiotic perturbation. Proc Natl Acad Sci USA. (2011) 108(Suppl. 1):4554-61. doi: 10.1073/pnas.10000 87107

78. Francino MP. Antibiotics and the human gut microbiome: dysbioses and accumulation of resistances. Front Microbiol. (2015) 6:1543. doi: 10.3389/fmicb.2015.01543

79. Geier B, Sogin EM, Michellod D, Janda M, Kompauer M, Spengler B, et al. Spatial metabolomics of in situ host-microbe interactions at the micrometre scale. Nat Microbiol. (2020) 5:498-510. doi: 10.1038/s41564-019-0 664-6

80. Faust K, Raes J. Microbial interactions: from networks to models. Nat Rev Microbiol. (2012) 10:538-50. doi: 10.1038/nrmicro2832

81. Hoffmann $\mathrm{KH}$, Rodriguez-Brito $\mathrm{B}$, Breitbart $\mathrm{M}$, Bangor $\mathrm{D}$, Angly F, Felts B, et al. Power law rank-abundance models for marine phage communities. FEMS Microbiol Lett. (2007) 273:224-8. doi: 10.1111/j.1574-6968.2007.00790.x

82. Zhou J, Deng Y, Luo F, He Z, Tu Q, Zhi X. Functional molecular ecological networks. mBio. (2010) 1:e00169-10. doi: 10.1128/mBio.00169-10

83. Dobzhansky T. Nothing in biology makes sense except in the light of evolution. Am Biol Teach. (1973) 35:125-9. doi: 10.2307/44 44260 
84. Callahan BJ, McMurdie PJ, Holmes SP. Exact sequence variants should replace operational taxonomic units in marker-gene data analysis. ISME J. (2017) 11:2639-43. doi: 10.1038/ismej.2017.119

85. Simonin M, Voss KA, Hassett BA, Rocca JD, Wang S-Y, Bier RL, et al. In search of microbial indicator taxa: shifts in stream bacterial communities along an urbanization gradient. Environ Microbiol. (2019) 21:3653-68. doi: 10.1111/1462-2920.14694

86. Gould AL, Zhang V, Lamberti L, Jones EW, Obadia B, Korasidis $\mathrm{N}$, et al. Microbiome interactions shape host fitness. Proc Natl Acad Sci USA. (2018) 115:E11951-60. doi: 10.1073/pnas.180934 9115
Conflict of Interest: The authors declare that the research was conducted in the absence of any commercial or financial relationships that could be construed as a potential conflict of interest.

Copyright (C) 2020 Duperron, Halary, Gallet and Marie. This is an open-access article distributed under the terms of the Creative Commons Attribution License (CC BY). The use, distribution or reproduction in other forums is permitted, provided the original author(s) and the copyright owner(s) are credited and that the original publication in this journal is cited, in accordance with accepted academic practice. No use, distribution or reproduction is permitted which does not comply with these terms. 